\title{
Mapping of polycrystalline films of biological fluids utilizing the Jones-matrix formalism
}

\author{
Vladimir A Ushenko ${ }^{1}$, Alexander V Dubolazovㄹ, Leonid Y Pidkamin ${ }^{1}$, \\ Michael Yu Sakchnovsky ${ }^{1}$, Anna B Bodnar ${ }^{1}$, Yuriy A Ushenko ${ }^{1}$, \\ Alexander G Ushenko ${ }^{1}$, Alexander Bykov ${ }^{2,3,4}$ and Igor Meglinski ${ }^{2,3,4,5,6}$ \\ 1 Yuriy Fedkovych Chernivtsi National University, 2 Kotsubinsky Str., 58012, Chernivtsi, Ukraine \\ 2 Opto-Electronics and Measurement Techniques, Faculty of Information Technology and Electrical \\ Engineering, University of Oulu, Erkki Koiso-Kanttilankatu 3, Oulu 90570, Finland \\ ${ }^{3}$ Interdisciplinary Laboratory of Biophotonics, National Research Tomsk State University, Prospect \\ Lenina 36, Tomsk 634050, Russia \\ ${ }^{4}$ ITMO University, Kronverkskiy Prospect 49, St Petersburg 197101, Russia \\ 5 Institute of Biology, Irkutsk State University, 3 Lenin St, Irkutsk 664003, Russia \\ 6 Institute of Engineering Physics for Biomedicine (PhysBio), National Research Nuclear University \\ MEPhI, 115409 Moscow, Russia
}

E-mail: igor.meglinski@oulu.fi

\begin{abstract}
Utilizing a polarized light approach, we reconstruct the spatial distribution of birefringence and optical activity in polycrystalline films of biological fluids. The Jones-matrix formalism is used for an accessible quantitative description of these types of optical anisotropy. We demonstrate that differentiation of polycrystalline films of biological fluids can be performed based on a statistical analysis of the distribution of rotation angles and phase shifts associated with the optical activity and birefringence, respectively. Finally, practical operational characteristics, such as sensitivity, specificity and accuracy of the Jones-matrix reconstruction of optical anisotropy, were identified with special emphasis on biomedical application, specifically for differentiation of bile films taken from healthy donors and from patients with cholelithiasis.
\end{abstract}

Keywords: scattering, polarization, tissue characterization, polarimetric imaging, Jones-matrix formalism, polarimetry

\section{Introduction}

The structure of biological tissues can be considered to be spatially inhomogeneous. In order to describe the transformation of polarized light in such complex media the most general approaches based on the Mueller matrix or Jones formalism have to be involved [1-3]. At present, there are many practical techniques based on measurement and analysis of Mueller matrices and/or the Jones vector of investigated depolarizing diffuse biological objects used in biological and medical research [4-7]. In recent times, laser polarimetry has been recognized as a powerful tool for screening thin (nondepolarizing) histological sections of biological tissues [8, 9]. An interconnection between the statistical moments of the first to fourth orders characterizing Mueller-matrix elements and the parameters of linear birefringence of biological tissues, such as fibrillary protein networks, has been established. This provides a unique possibility for the diagnosis of cancerrelated abnormalities in biological tissues [8-13].

Nevertheless, the various mechanisms of optical anisotropy of biological tissues/layers $[14,15]$ and the presence of a variety of optically anisotropic biological liquids (including 


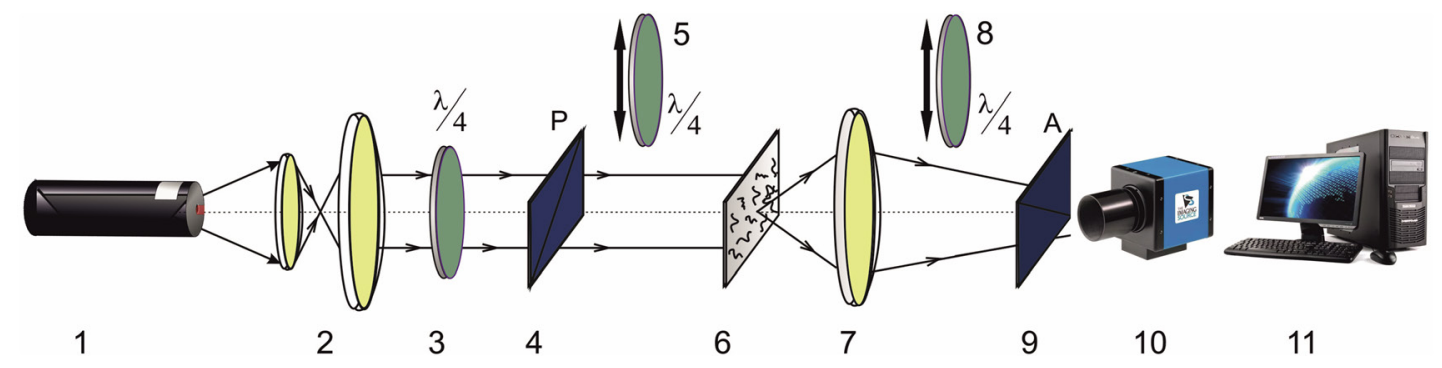

Figure 1. Schematic diagram of the experimental setup: 1 -He-Ne laser; 2 - collimator; 3 -quarter-wave plate; 5 , 8-additional quarterwave plates; 4, 9—-polarizer and analyzer, respectively; 6-tissue sample; 7—optical lens; 10 -CCD camera; 11—computer; see more details in the text.

blood, urine, bile, saliva, etc), for which laser polarimetry techniques have not been used much $[16,17]$, mean that further development of laser polarimetry is required. Besides, the Jones-matrix formalism is more appropriate for describing the transformation of non-depolarizing objects in terms of their diagnosis by polarized laser light.

There is also growing interest in dislocations of phase singularities [18], and in solution of the inverse problem, i.e. reconstruction of the spatial distribution of the phase of polarized light that has propagated through optically anisotropic, polycrystalline, optically thin biological layers [19, 20].

In the current study in the framework of a generalization of the polarization-based approach for diagnosis of optically anisotropic thin layers (more specifically bile films), we are focused on the development of 2D polarization-phase reconstruction of anisotropy of polycrystalline networks with the final aim of early diagnosis of cholelithiasis. This disease occurs latently and can be diagnosed only in quite late stages, when the crystalline concretions have already been formed. The World Health Organization has reported that a number of people in the world suffer seriously from cholelithiasis.

\section{Materials and method}

Based on the approach developed previously [15, 16], we utilize the phase anisotropy, namely optical activity and linear birefringence, of the polycrystalline structure of films of biological fluids. The basic theory of phase anisotropy of such polycrystalline films is used to interpret the experimental data.

The Jones-matrix formalism is utilized to study the propagation of laser waves through a thin, optically anisotropic medium. In this approach the optically thin film is represented as a series of $N$ layers with a phase anisotropy associated with linear $(\delta)$ and/or circular $(\theta)$ birefringence. The corresponding Jones matrix of an optically thin layer with phase anisotropy $\{J\}$ can be presented as [8]

$$
\{J\}=\{R(\zeta)\}=\left\|\begin{array}{cc}
\cos U-\mathrm{i} \frac{\delta \sin U}{2 U} & -\zeta \frac{\sin U}{U} \\
\zeta \frac{\sin U}{U} & \cos U+\mathrm{i} \frac{\delta \sin U}{2 U}
\end{array}\right\|,
$$

where $U=\left(\theta^{2}+0.25 \delta^{2}\right)^{0.5}$.

Gerard [21] suggested another notation for Jones matrices, when matrix $\{J\}$ is written as real $R_{i k}$ (modulus) and imaginary $\Theta_{i k}$ (phase angles) components:

$$
\{J\}=\left\|\begin{array}{ll}
R_{11}(r) \exp \Theta_{11}(r) & R_{12}(r) \exp \Theta_{12}(r) \\
R_{21}(r) \exp \Theta_{21}(r) & R_{22}(r) \exp \Theta_{22}(r)
\end{array}\right\| .
$$

Comparing expressions (1) and (2) enables us to determine the interrelations between them:

$$
\begin{gathered}
R_{11}=R_{22}=\sqrt{\cos ^{2} U+0.25 \delta^{2} \frac{\sin ^{2} U}{U^{2}}}, \\
\Theta_{11}=-\Theta_{22}=\arctan \left(\frac{\delta}{2 U} \tan U\right), \Theta_{12}=\Theta_{21}=0, \\
R_{12}=R_{21}=\zeta \frac{\sin U}{U},
\end{gathered}
$$

and on this basis to obtain the algorithm for reproduction of the polarization of the phase anisotropy parameters for the optically thin birefringent layer:

$$
\begin{aligned}
& \delta=\frac{2 \arccos \left(R_{11} \cos \Theta_{11}+R_{22} \cos \Theta_{22}\right)}{1+\frac{R_{12} \cos \Theta_{12}-R_{21} \cos \Theta_{21}}{R_{22} \cos \Theta_{22}-R_{11} \cos \Theta_{11}},} \\
& \theta=\frac{2 \arccos \left(R_{11} \cos \Theta_{11}+R_{22} \cos \Theta_{22}\right)}{1+\frac{R_{22} \cos \Theta_{22}-R_{11} \cos \Theta_{11}}{R_{12} \cos \Theta_{22}-R_{21} \cos \Theta_{11}}} .
\end{aligned}
$$

The measurements of coordinate distributions of the Jonesmatrix elements were performed in the experimental setup presented schematically in figure 1 .

The bile film samples were illuminated by the parallel (diameter $\left.\varnothing=2 \times 10^{3} \mu \mathrm{m}\right)$ weak $(W=5 \mathrm{~mW})$ beam 1 of a He-Ne laser $(\lambda=0.6328 \mu \mathrm{m})$. The use of a collimated illuminating beam provides the same conditions of transformation of the polarization states at different points of the illuminated area of the object. This allows comparative studies between different groups of samples. The polarization light source consisted of a quarter-wave plate 3 and polarizer 4 . The image of the samples 6 was projected into the light-sensitive plane of a CCD camera 10 (The Imaging Source DMK 41AU02. AS, monochrome 1/2" CCD, Sony ICX205AL (progressive scan); resolution-1280 $\times 960$; sensitivity- $0.051 x$; dynamic range -8 bit; SNR -9 bit) by means of optical system 7 . In this experimental arrangement 7 designates an image-forming apparatus, which consists of a strain-free objective (Nikon CFI Achromat P, working distance- $3.0 \mathrm{~mm}$, focal distance- $50 \mathrm{~mm}, \mathrm{NA}-0.1$, magnification-4×) and a tube lens (focal distance $200 \mathrm{~mm}$ ). Polarization analysis of the sample 

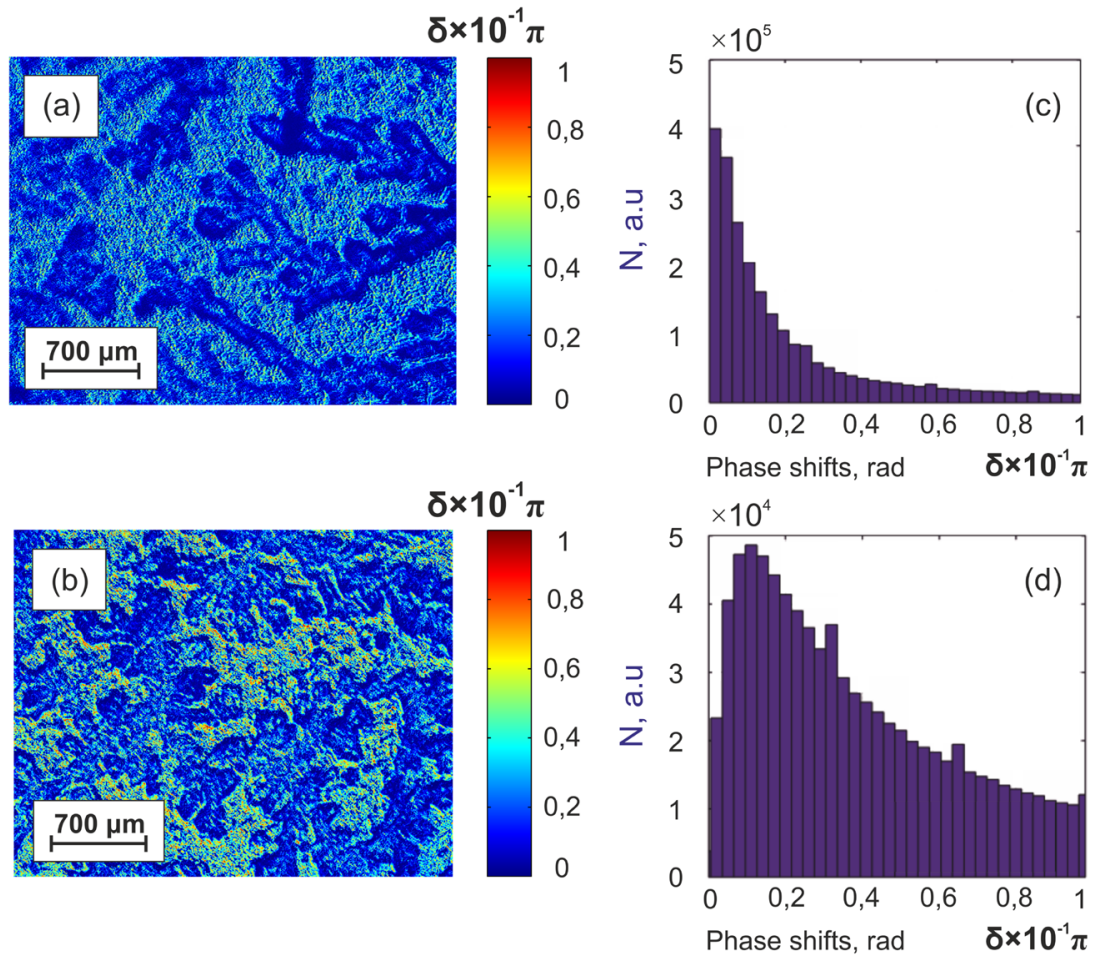

Figure 2. Spatial intensity distributions (a) and (b) and the histograms of corresponding values of the phase shifts $\delta$ (c) and (d), formed by polycrystalline film of bile taken from healthy volunteers (a) and (c) and patients with cholelithiasis (b) and (d).

images was performed by means of quarter-wave plate 8 and polarizer-analyzer 9 . The values of $R_{i k}$ and $\Theta_{i k}$ were measured using the classical technique described in Gerrard's book [21].

The distributions of polarization states in this image are uniquely associated with the distribution phase anisotropy parameters, because the manifestations of dichroism or diattenuation in the 'red' region of the spectrum are minimal. This allows solution of the inverse problem - the reconstruction of the distribution of values of the phase shift between the orthogonal components of the amplitude of the polarized laser radiation. The experimental implementation of the criterion of single-scattering mode is the value of the extinction coefficient $\tau$, which can be measured on the basis of the Beer-LambertBouguer law. For bile films the ratio between the intensities of the incident and transmitted forward laser radiation lies in the range $0.92 \leqslant I / I_{0} \leqslant 0.98 \leftrightarrow 0.087 \leqslant \tau \leqslant 0.098$. On the basis of equations (6) and (7), the parameters of phase $(q \equiv \theta, \delta)$ anisotropy were found for each pixel of the CCD camera. For objective assessment of $N$ histograms of distributions $q \equiv \theta, \delta$ the set of statistical moments of the first to fourth orders (mean $M_{1}$, dispersion $M_{2}$, skewness $M_{3}$ and kurtosis $M_{4}$ ) was determined in a similar way to that suggested in $[8,9]$.

\section{Results and discussion}

Figures 2 and 3 present the results from the reconstruction of the Jones-matrix parameters of polycrystalline bile films. The figures consist of spatial intensity distributions (see parts (a) and (b) in figures 2 and 3) and the histograms (see parts (c) and (d)) for two arbitrarily chosen samples of healthy donors (group 1, see parts (a) and (c) in figures 2 and 3) and patients suffering from cholelithiasis (group 2, parts (b) and (d) figures 2 and 3). A comparative analysis of the data in these figures showed similar tendencies for the investigated samples.

It was determined that for the bile film taken from a donor (see figure 2(a)), the value of linear birefringence is sufficiently small if compared to the sample (see figure 2(b)) from cholelithiasis patients. The main extremes of histograms $N(\delta)$ of group 1 (see figure 2(c)) are localized in the area from $\delta=0.1 \times 10^{-1} \pi$ to $\delta=0.13 \times 10^{-1} \pi$. For group 2 (see figure $2(\mathrm{~d})$ ) bigger values of phase shifts, $\delta=0.32 \times 10^{-1} \pi$ to $\delta=0.41 \times 10^{-1} \pi$, are the most probable.

Thus, the increases in mean $\left(M_{1} \uparrow\right)$ and dispersion $\left(M_{2} \uparrow\right)$ of histograms $N(\delta)$ appears to be a statistical indicator of the cholelithiasis condition. In that case the statistical moments of higher orders (skewness $\left(M_{3} \downarrow\right)$ and kurtosis $\left.\left(M_{4} \downarrow\right)\right)$ decrease. Physically, the obtained results can be related to the known data from biochemical analysis - a greater concentration of the liquid-crystalline fraction consisting of the set of liquid acicular crystals of fatty acids and cholesterol monohydrate in the bile film from cholelithiasis patients.

The similar tendencies to those in $\delta$ are determined in the statistical changes of parameter $\theta$ (see figure 3 ), characterizing the optical activity of spherulitic crystals of calcium bilirubinate in bile films. Due to the increase in concentration of such crystals in the bile of cholelithiasis patients, the probability of greater values of $\theta$, formed by the sample of group 2, increases (see figure 3(d)). Quantitatively this illustrates the increase in the probability of forming greater values $\theta=0.7 \times 10^{-1} \pi$ to $\theta=0.95 \times 10^{-1} \pi$ in distributions $N(\theta)$ (see figure $3(\mathrm{~d})$ ). In other words, the 

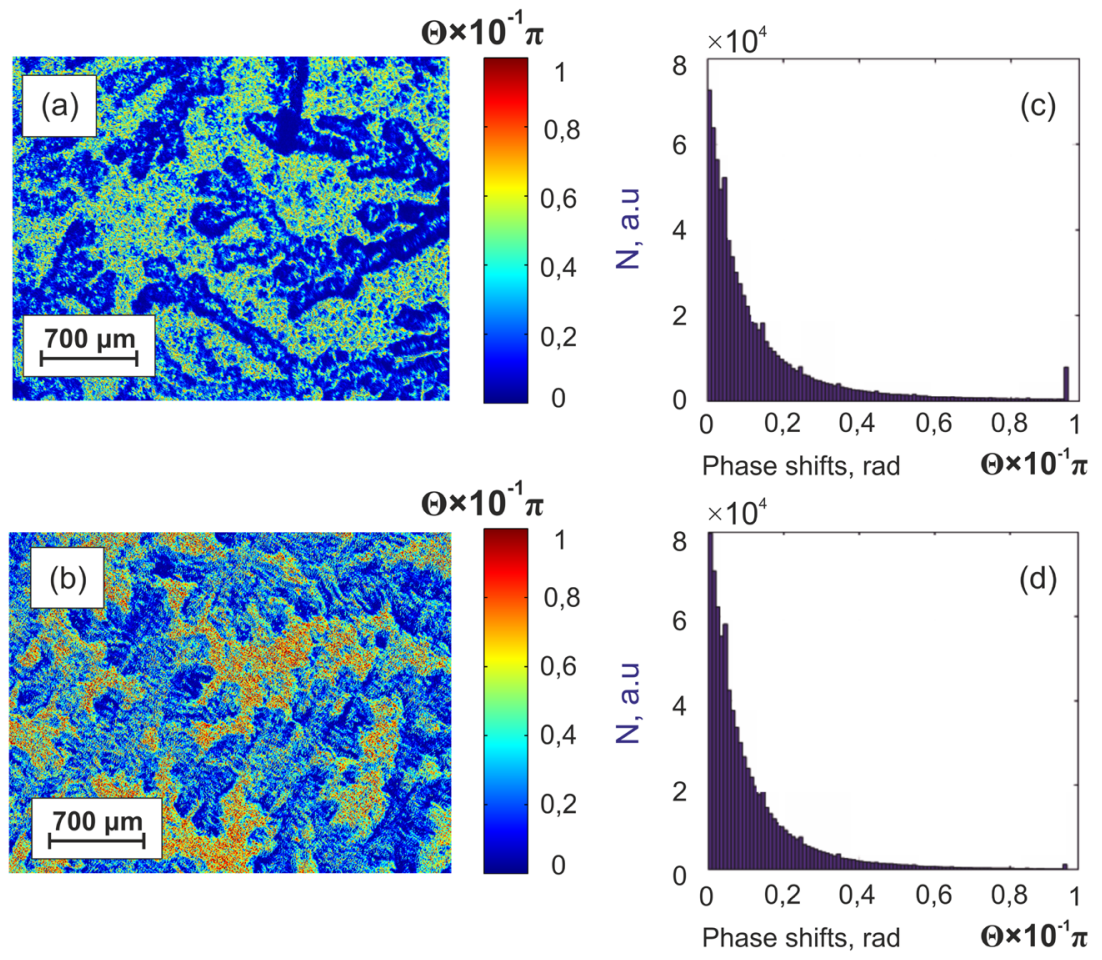

Figure 3. Spatial intensity distributions (a) and (b) and the histograms of corresponding values of the phase shifts $\theta$ (c) and (d), formed by polycrystalline film of bile taken from healthy volunteers (a) and (c) and patients with cholelithiasis (b) and (d).

Table 1. Average $\left(\bar{M}_{i=1 ; 2 ; 3 ; 4}\right)$ and standard deviations $( \pm \sigma)$ of statistical moments $M_{i=1 ; 2 ; 3 ; 4}$ of optical anisotropy distributions of bile films of groups 1 and 2, both counted for 57 subjects.

\begin{tabular}{llllll}
\hline & \multicolumn{2}{c}{$\delta(n=57)$} & & \multicolumn{2}{c}{$\theta(n=57)$} \\
\cline { 2 - 3 } \cline { 5 - 6 }$q$ & Group 1 & Group 2 & & Group 1 & Group 2 \\
\hline$M_{1}$ & $0.13 \pm 0.009$ & $0.17 \pm 0.012$ & & $0.09 \pm 0.007$ & $0.12 \pm 0.009$ \\
$M_{2}$ & $0.16 \pm 0.012$ & $0.23 \pm 0.018$ & & $0.13 \pm 0.0099$ & $0.19 \pm 0.015$ \\
$M_{3}$ & $0.78 \pm 0.056$ & $0.39 \pm 0.031$ & & $0.92 \pm 0.078$ & $0.51 \pm 0.039$ \\
$M_{4}$ & $0.91 \pm 0.081$ & $0.56 \pm 0.041$ & & $1.13 \pm 0.11$ & $0.67 \pm 0.052$ \\
\hline
\end{tabular}

following statistical scenario is realized for the pathological state: $M_{1}(\theta) \uparrow ; \quad M_{2}(\theta) \uparrow ; \quad M_{3}(\theta) \downarrow ; \quad M_{4}(\theta) \downarrow$.

For the possible clinical application of both methods the following were determined for each group of samples [22-24]: (i) average (within group 1 and group 2) values of statistical moments $M_{i=1 ; 2 ; 3 ; 4}(q)$, their standard deviations $\pm \sigma$ and histograms $M\left(R_{i}\right)$ (table 1); (ii) as is traditional for the operational characteristics of probative medicine-sensitivity $\left(S e=\frac{a}{a+b} \times 100 \%\right)$, specificity $\left(S p=\frac{c}{c+d} \times 100 \%\right)$ and balanced accuracy $\left(A c=\frac{S_{e}+S_{p}}{2}\right)$, where $a$ and $b$ are the number of correct and wrong diagnoses within group 2; $c$ and $d$ are the same within group 1 (table 2).

Differentiation of polarization-phase tomograms of bile layers was performed by cross-sectional comparison of distribution histograms $M_{i=1 ; 2 ; 3 ; 4}(q)$. If the mean value of some moment $\bar{M}_{i}(q)$ in the test group 1 is not within the standard deviation $\sigma$ of the investigated group 2, the difference between
Table 2. Operational characteristics of the method of Jones-matrix reconstruction of the polycrystalline structure of bile films.

\begin{tabular}{llll}
\hline$q$ & $M_{i}$ & $\delta$ & $\theta$ \\
\hline$A c\left(Z_{i}\right)$ & $M_{1}$ & $76 \%$ & $78 \%$ \\
& $M_{2}$ & $85 \%$ & $87 \%$ \\
& $M_{3}$ & $96 \%$ & $90 \%$ \\
& $M_{4}$ & $93 \%$ & $94 \%$ \\
\hline
\end{tabular}

moments $\bar{M}_{i}(q)$ is considered statistically reliable. Then the analysis of the region of overlap of histograms $M_{i=1 ; 2 ; 3 ; 4}(q)$ that determine the sensitivity $S e$, specificity $S p$ and accuracy Ac appears to be relevant.

The comparative analysis of the data obtained (see table 1) showed that the differences between the values of average $\bar{M}_{i=1 ; 2 ; 3 ; 4}(q)$ moments of all orders are statistically reliable. However, there is an intergroup overlap for all histograms $N\left(M_{i}\right)$. Moreover, the range of such an overlap is inversely proportional to the value of the difference between the averages $\bar{M}_{i=1 ; 2 ; 3 ; 4}(q)$. The following quantitative differences between average statistical moments $\bar{M}_{i}(q)$ are determined:

(i) Linear birefringence for Jones-matrix-reconstructed distributions $\delta$ of polycrystalline bile films of both types; the difference between statistical moments $\bar{M}_{i=1 ; 2 ; 3 ; 4}(\delta)$ is $\left\{\Delta M_{1}(\delta)=1.31 ; \Delta M_{2}(\delta)=1.43 ; \Delta M_{3}(\delta)=2.0 ; \Delta M_{4}(\delta)=1.63\right\}$.

(ii) Circular birefringence for statistical moments $\bar{M}_{i=1 ; 2 ; 3 ; 4}(q)$ characterizing the distribution $\theta$, formed by optically active structures of bile film; the following differences are determined: $\left\{\Delta M_{1}(\theta)=1.33\right.$; 
$\left.\Delta M_{2}(\theta)=1.46 ; \Delta M_{3}(\theta)=1.8 ; \Delta M_{4}(\theta)=1.69\right\}$. As the data presented show, the statistical moments of the third and fourth orders characterizing the histograms $N(q)$ of the bile films of both groups of patients prove to be the most sensitive. On the other hand, the greater $\Delta M_{i=1 ; 2 ; 3 ; 4}(q)$ is, the more informative $\left(S_{\mathrm{e}} \uparrow, S_{\mathrm{p}} \uparrow, A_{\mathrm{c}} \uparrow\right)$ the method appears to be.

Table 2 presents the parameters of the polarization-phase method of Jones-matrix reconstruction of the phase anisotropy of polycrystalline films of bile.

The comparative analysis of operational characteristics of the method of Jones-matrix polarization reconstruction of the polycrystalline structure of bile films revealed clinically optimal parameters (those for $M_{3}$ and $M_{4}$ ):

$$
\left\{\begin{array}{l}
\delta \rightarrow M(\delta) \equiv\left\{A c\left(R_{3 ; 4}\right)=93 \%-96 \%\right\} \\
\theta \rightarrow M(\theta) \equiv\left\{A c\left(R_{3 ; 4}\right)=90 \%-94 \%\right\}
\end{array}\right.
$$

The obtained results enable us to state a rather high level of accuracy of Jones-matrix polarization-phase tomography. According to the criteria of probative medicine [24] the parameters $M(\delta, \theta) \sim 90 \%-96 \%$ correspond to high quality.

\section{Conclusion}

To sum up, the model of generalized optical anisotropy and the technique of Jones-matrix reconstruction of the optical anisotropy parameters of polycrystalline bile films have been introduced. On this basis the Jones-matrix algorithms describing the polarization nature of partial mechanisms of optical anisotropy of biological fluids were defined. By means of statistical analysis the interconnection between the first- to fourth-order statistical moments of the anisotropy parameters of bile films and the changes in the structure of bile in healthy people and in cholelithiasis patients were determined. The efficiency has been proved of Jones-matrix reconstruction of the optical anisotropy parameters of bile films in diagnostics of the early stages of cholelithiasis.

\section{Acknowledgments}

This work was supported by the Strategic Action funding programme of the ITEE Faculty of the University of Oulu. IM also acknowledges partial support from the MEPhI Academic Excellence Project (Contract No. 02.a03.21.0005) and Russian Science Foundation (Grant No.: 15-14-10008).

\section{References}

[1] Tuchin V V, Wang L and Zimnyakov D A 2006 Optical Polarization in Biomedical Applications (New York: Springer)

[2] Bickel W S and Bailey W M 1985 Stokes vectors, Mueller matrices, and polarization of scattered light Am. J. Phys. $\mathbf{5 3} 468-78$
[3] Chipman R A 2010 Polarimetry Handbook of Optics: Vol. I-Geometrical and Physical Optics, Polarized Light, Components and Instruments ed M Bass (New York: McGraw-Hill) pp 22.1-22.37

[4] Ghosh N, Wood M and Vitkin A 2010 Polarized light assessment of complex turbid media such as biological tissues using Mueller matrix decomposition Handbook of Photonics for Biomedical Science ed V V Tuchin (London: CRC Press) pp 253-82

[5] Novikova T et al 2017 Optical biopsy of tissue with Mueller polarimetry: theory and experiments Proc. SPIE $10060100600 \mathrm{~V}$

[6] Novikova T, Meglinski I, Ramella-Roman J C and Tuchin V V 2016 Polarized light for biomedical applications J. Biomed. Opt. 21071001

[7] Kunnen B, Macdonald C, Doronin A, Jacques S, Eccles M and Meglinski I 2015 Application of circularly polarized light for non-invasive diagnosis of cancerous tissues and turbid tissue-like scattering media J. Biophoton. $8317-23$

[8] Angelsky O V, Ushenko A G, Ushenko Yu A, Pishak V P and Peresunko A P 2010 Statistical, correlation and topological approaches in diagnostics of the structure and physiological state of birefringent biological tissues Handbook of Photonics for Biomedical Science ed V Tuchin (Boca Raton, FL: CRC Press) pp 283-322

[9] Ushenko Y A, Boychuk T M, Bachynsky V T and Mincer O P 2013 Diagnostics of structure and physiological state of birefringent biological tissues: statistical, correlation and topological approaches Handbook of Coherent-Domain Optical Methods ed V Tuchin (New York: Springer) pp 107-48

[10] Ghosh N and Vitkin I A 2011 Tissue polarimetry: concepts, challenges, applications and outlook J. Biomed. Opt. 16110801

[11] Jacques S L 2011 Polarized light imaging of biological tissues Handbook of Biomedical Optics ed D Boas et al (Boca Raton, FL: CRC Press) pp 649-69

[12] Layden D, Ghosh N and Vitkin A 2013 Quantitative polarimetry for tissue characterization and diagnosis Advanced Biophotonics: Tissue Optical Sectioning ed R K Wang and V V Tuchin (Boca Raton, FL: CRC Press) pp 73-108

[13] Vitkin A, Ghosh N and de Martino A 2015 Tissue polarimetry Photonics: Scientific Foundations, Technology and Applications vol 4, ed D L Andrews (New Jersey: Wiley) pp 239-321

[14] Ushenko V A and Gorsky M P 2013 Complex degree of mutual anisotropy of linear birefringence and optical activity of biological tissues in diagnostics of prostate cancer Opt. Spectrosc. 115 290-7

[15] Ushenko V A and Gavrylyak M S 2013 Azimuthally invariant Mueller-matrix mapping of biological tissue in differential diagnosis of mechanisms protein molecules networks anisotropy Proc. SPIE $\mathbf{8 8 1 2} 88120$ Y

[16] Ushenko Yu A, Ushenko V A, Dubolazov A V, Balanetskaya V O and Zabolotna N I 2012 Mueller-matrix diagnostics of optical properties of polycrystalline networks of human blood plasma Opt. Spectrosc. $112884-92$

[17] Ushenko Yu A, Dubolazov A V, Balanetskaya V O, Karachevtsev A O and Ushenko V A 2012 Wavelet-analysis of polarization maps of human blood plasma Opt. Spectrosc. 113 332-43

[18] De Angelis L, Alpeggiani F, Di Falco A and Kuipers L 2016 Spatial distribution of phase singularities in optical random vector waves Phys. Rev. Lett. 117093901 
[19] Ungurian V P, Ivashchuk O I and Ushenko V A 2011 Statistical analysis of polarizing maps of blood plasma laser images for the diagnostics of malignant formations Proc. SPIE 8338 83381L

[20] Ushenko V A, Dubolazov O V and Karachevtsev A O 2014 Two wavelength Mueller matrix reconstruction of blood plasma films polycrystalline structure in diagnostics of breast cancer Appl. Opt. 53 B128-39
[21] Gerrard A and Burch A J 2012 Introduction to Matrix Methods in Optics (New York: Dover)

[22] Cassidy L 2005 Basic concepts of statistical analysis for surgical research J. Surg. Res. 128 199-206

[23] Davis C S 2002 Statistical Methods of the Analysis of Repeated Measurements (New York: Springer)

[24] Petrie A and Sabin B 2005 Medical Statistics at a Glance (Oxford: Blackwell) 